\section{Modulateurs du métabolisme du cholestérol et avenir du traitement de l'athérosclérose}

Svetlana Morozova, Isabelle Suc-Royer, Johan Auwerx maladies, ce qui représente près d'un tiers de tous les décès. Dans les pays industrialisés, l'athérosclérose, responsable d'environ $50 \%$ des décès, est la première cause de mortalité. Les traitements actuels de cette maladie visent à en maîtriser les facteurs de risque, dont l'un des principaux est le taux élevé de cholestérol plasmatique. Les médicaments les plus efficaces contre l'athérosclérose sont hypocholestérolémiants et appartiennent à la classe des statines. Cependant, les statines diminuent la mortalité due aux maladies cardiovasculaires chez seulement un tiers des patients. De nouveaux produits, susceptibles de diminuer la cholestérolémie ou de ralentir la formation des lésions au niveau de la paroi vasculaire, devraient être développés. <
L'athérosclérose est caractérisée par la formation de plaques d'athérome constituant des sites inflammatoires dans la paroi artérielle avec accumulation de lipides et d'éléments fibreux (Figure 1) [1-3]. L’hypercholestérolémie est l'un des principaux facteurs de risque de la maladie. L'incidence des accidents coronariens est directement liée au taux plasmatique de cholestérol-LDL (low density lipoprotein) et inversement liée au taux de cholestérol-HDL (high density lipoprotein) $[4,5]$. Ainsi, le métabolisme du cholestérol (Figures 2 et 3 ) est un élément clé du développement des maladies cardiovasculaires $[6,7]$.

Les dyslipidémies étant une des causes majeures de l'athérosclérose, les principaux médicaments utilisés visent à les corriger. Trois types de médicaments sont disponibles (les statines, les fibrates, les résines), auxquels s'ajoutent trois produits, la niacine, le probucol, et l'ézétimibe (Figure 3). L'effet hypocholestérolémiant des statines est dû à la diminution de la biosynthèse du cholestérol par inhibition

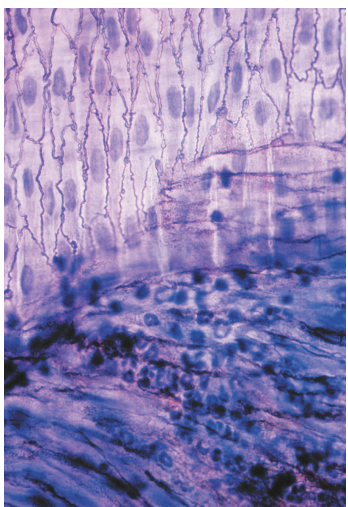

S. Morozova, I. Suc-Royer: Fournier Pharma, Département Biologie exploratoire, 50, rue de Dijon, 21121 Daix, France. smorozova@aol.com J. Auwerx: Institut de Génétique et de biologie moléculaire et cellulaire (IGBMC) 1, rue Laurent Fries, Parc de l'enzyme 3-hydroxy3-méthylglutaryl coend'innovation, 67404 Illkirch, France.

réductase. Ces molécules médicamenteuses ont de plus des effets anti-athérogènes sur la paroi vasculaire (antiinflammatoire, stabilisation de la plaque) [8-10]. La combinaison de ces effets des statines peut être à l'origine de leur efficacité démontrée par des études cliniques: diminution d'environ $30 \%$ du nombre d'événements cardiovasculaires et de décès [11]. Cette efficacité en fait des médicaments de choix pour la prévention de l'athérosclérose. L'effet principal des fibrates réside dans la diminution du taux des triglycérides plasmatiques. Ce sont des agonistes du peroxisome proliferator activated receptor $\alpha$ (PPAR $\alpha$ ) conduisant à l'activation de la lipoprotéine lipase et l'augmentation des apolipoprotéines Al et All, constituants majeurs des HDL [12]. Les résines favorisent une élimination accrue des acides biliaires dans les fèces, ce qui augmente leur synthèse à partir du cholestérol. La niacine et le probucol sont peu utilisés, notamment en raison d'une mauvaise tolérance ou d'une faible efficacité [9]. L'ézétimibe, un produit hypocholestérolémiant commercialisé pour la première fois aux États-Unis en novembre 2002, est un inhibiteur spécifique de l'absorption intestinale du cholestérol (Figure 3). II agirait sur le/les transporteur(s) de 
cholestérol dans les entérocytes. II s'impose avant tout comme un complément aux statines et permet une réduction supplémentaire de la cholestérolémie de 15-20\% par rapport à l'utilisation d'une statine seule [13, 14].

Parmi les produits non ciblés sur le métabolisme lipidique, il faut noter les inhibiteurs de l'enzyme de conversion de l'angiotensine (hypotenseurs) qui exercent une action anti-inflammatoire et réduisent le nombre d'événements cardiovasculaires [15].

Le présent article étant axé sur les futurs médicaments contre l'athérosclérose, le lecteur trouvera plus d'informations sur les médicaments existants dans les publications citées. amélioré par l'utilisation de produits qui agissent directement sur la paroi vasculaire en empêchant la formation des lésions. D'un intérêt thérapeutique notable, ces produits anti-athéromateux n'existent pas encore aujourd'hui dans la pratique médicale, mais sont en cours de développement.

\section{Nouveaux médicaments en développement}

La recherche de nouveaux agents thérapeutiques est fondée sur la modification de l'activité d'une cible moléculaire précise. Pour certaines cibles du métabolisme du cholestérol, des molécules médicamenteuses

\section{Pourquoi de nouveaux médicaments contre l'athérosclérose?}

Des études épidémiologiques et cliniques indiquent l'existence d'une relation directe et continue entre la cholestérolémie et le risque cardiovasculaire. Différentes observations suggèrent qu'il n'existe pas de seuil de cholestérol-LDL en dessous duquel une réduction supplémentaire ne produirait pas de bénéfices sur ce risque. Ainsi, la notion du lower is better associée aux taux recommandés de cholestérol-LDL à atteindre chez les patients implique de réfléchir à la façon de réduire plus efficacement ces taux [16]. Cette réduction peut être atteinte en augmentant les doses de statines. Ces doses élevées pouvant être toxiques, il serait plus bénéfique de combiner des doses modérées de statines avec des agents hypocholestérolémiants possédant de nouveaux mécanismes d'action sur le métabolisme lipidique. Le traitement de l'athérosclérose pourrait être

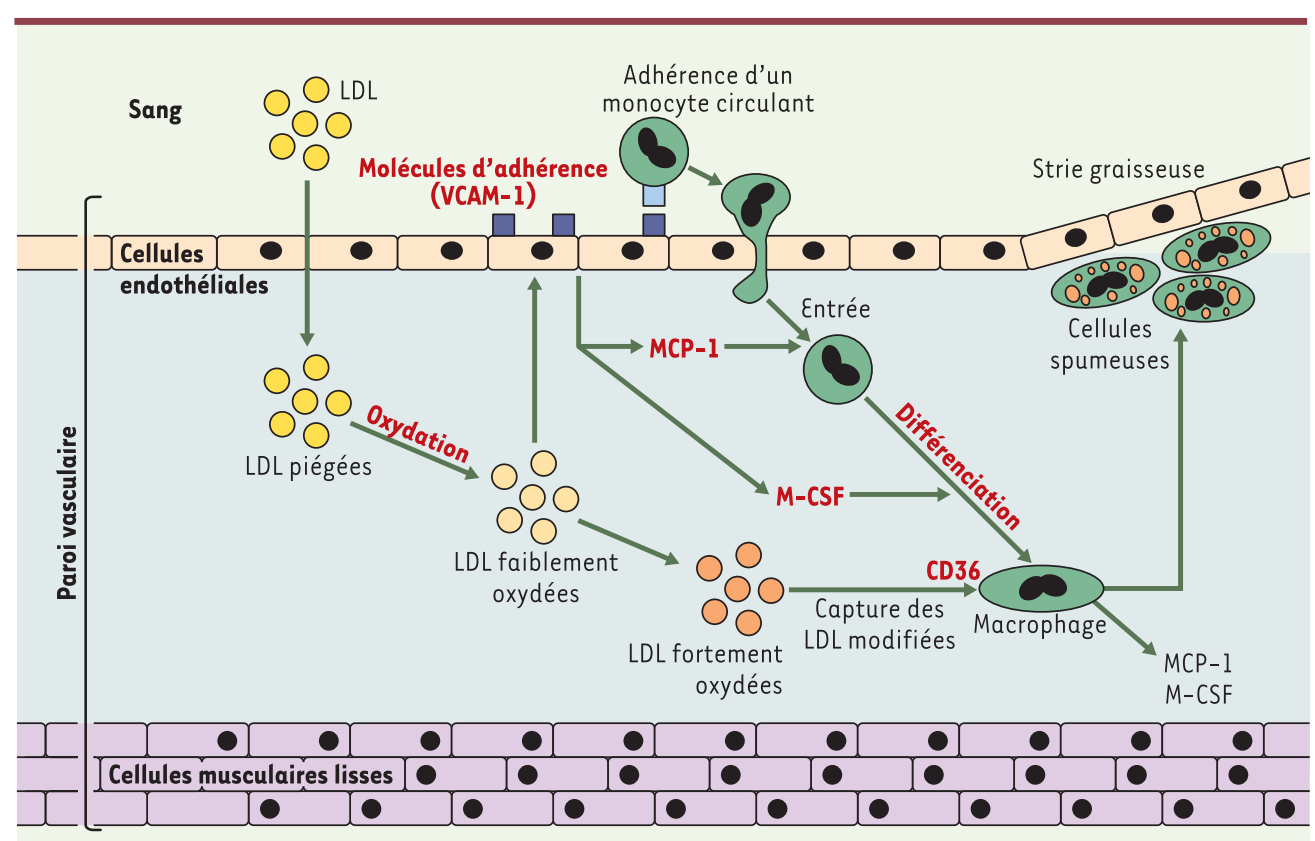

Figure 1. Formation d'une lésion athéromateuse. Les sites privilégiés de formation des lésions sont les embranchements des vaisseaux qui correspondent aux zones de turbulence du courant sanguin. Dans ces zones, les forces hémodynamiques changent les propriétés de l'endothélium en augmentant notamment sa perméabilité aux LDL (low density lipoprotein) circulantes. Les LDL diffusent dans la paroi vasculaire où elles se trouvent piégées dans le réseau de fibres et subissent des modifications oxydatives. Les LDL faiblement oxydées ainsi formées ont des propriétés pro-inflammatoires: elles induisent dans les cellules endothéliales la production de molécules d'adhérence (VCAM-1: vascular cell adhesion molecule-1), de facteurs chimio-attractants (MCP1: monocyte chemoattractant protein 1) et de facteurs de différenciation (M-CSF: macrophage colony stimulating factor-1). Ces modifications dans les cellules endothéliales permettent aux monocytes circulants d'adhérer à la paroi vasculaire, d'y pénétrer et de s'y différencier en macrophages résidents. La production de MCPl et de M-CSF par ces macrophages maintient un état d'inflammation chronique dans la lésion. Les LDL continuent à subir des modifications et sont transformées en LDL fortement oxydées qui sont reconnues par les récepteurs « éboueurs » (scavengers) des macrophages, dont le CD36 (cluster of differenciation 36). La capture des LDL oxydées par ces récepteurs n'étant pas contrôlée, les macrophages accumulent des lipides et se transforment en cellules spumeuses, qui sont à l'origine de la lésion précoce du type strie graisseuse, élément clé du processus d'athérogenèse. La lésion évolue ensuite successivement vers les stades fibreux et complexe et peut aboutir à la rupture qui provoquera la formation d'un thrombus occluant la lumière du vaisseau sanguin. Les cibles moléculaires ou les processus qu'il faut inhiber pour ralentir le développement des lésions athéromateuses sont indiqués en rouge. 
ont déjà été identifiées et sont en cours de développement préclinique ou clinique [17-20] (Figure 3).

\section{Inhibiteurs de squalène synthase}

L'acétate est le précurseur de molécules stéroïdes et non stéroïdes (Figure 4). L'inhibition de l'HMG CoA réductase peut provoquer des effets secondaires dus au déficit de produits non stéroïdes. Les médicaments qui agiraient spécifiquement sur la voie de synthèse des produits stéroïdes n'induiraient pas de tels effets, tout en baissant la cholestérolémie. Ainsi, les inhibiteurs de squalène synthase, enzyme catalysant la première étape de synthèse des stérols, ont été étudiés [21]. Cependant, l'accumulation du farnésyl pyrophosphate consécutive à cette inhibition s'est révélée toxique chez l'animal. De plus, l'inhibition des étapes finales de synthèse du cholestérol pourrait avoir des conséquences graves au vu des études réalisées avec le triparanol $^{1}$ chez l'animal [22]. Ainsi, la prudence s'impose concernant les inhibiteurs de synthèse du cholestérol, ce qui pourrait expliquer le faible nombre d'inhibiteurs de squalène synthase en développement clinique [20].

\section{Inhibiteurs de MTP}

La MTP (microsomal transfer protein) joue un rôle majeur dans l'assemblage des chylomicrons intestinaux et des VLDL (very low density lipoprotein) hépatiques (Figure 3). Son inhibition permet de diminuer la sécrétion des chylomicrons et donc le passage des lipides, dont le cholestérol,

${ }^{1}$ Inhibiteur de la desmostrérol réductase, enzyme catalysant la réaction conduisant du 7-déshydrodesmostérol au 7-déshydrocholestérol, précurseur direct du cholestérol.

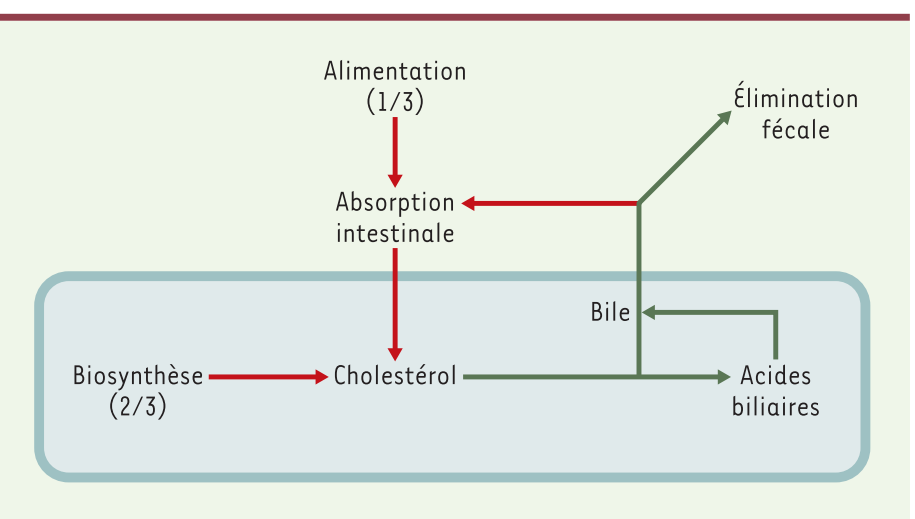

Figure 2. Voies d'entrée (en rouge) et de sortie (en vert) du cholestérol de l'organisme. Le cholestérol peut provenir de l'alimentation, qui est à l'origine d'environ un tiers des apports journaliers, ou de la biosynthèse, responsable de l'apport des deux tiers restants. La seule voie importante d'élimination du cholestérol est sa transformation hépatique en acides biliaires. Le foie excrète le cholestérol et les acides biliaires dans la bile qui est déversée dans la lumière intestinale au moment de la prise alimentaire. La fraction non (ré)absorbée d'acides biliaires et de cholestérol est éliminée dans les fèces. à travers la barrière intestinale [7]. De plus, cette inhibition permet de réduire la production des VLDL et, par conséquent, du cholestérol-LDL plasmatique. L'effet hypocholestérolémiant des inhibiteurs de MTP a été montré dans des modèles animaux. Certains de ces inhibiteurs sont entrés en phase de développement clinique [20, 23, 24]. Cependant, à l'heure actuelle, on ne sait pas si ces produits peuvent être utilisés en pratique médicale en raison d'une accumulation possible de triglycérides dans des organes cibles comme le foie.

\section{Inhibiteurs d'ACAT}

L'enzyme ACAT (acyl-coenzyme A-cholesterol acyltransferase) catalyse l'estérification du cholestérol tissulaire et favorise son stockage dans les cellules. Elle existe sous deux formes, ACATl, qui participe à la formation des cellules spumeuses dans la paroi vasculaire, et ACAT2, impliquée dans la formation des chylomicrons dans l'intestin et des VLDL dans le foie (Figure 3). L'inhibition de l'ACATl préviendrait la formation des plaques d'athérome, tandis que celle de l'ACAT2 conduirait à une diminution d'absorption intestinale du cholestérol [7] et à un effet hypocholestérolémiant. Les inhibiteurs d'ACAT identifiés jusqu'à présent sont non sélectifs et inhibent les deux formes de l'enzyme. L'un d'entre eux, l'avasimibe (Pfizer), dont les activités hypocholestérolémiante et anti-athéromateuse ont été montrées chez l'animal [25], connaît le développement clinique le plus avancé (phase III) [20]. L'avenir des inhibiteurs d'ACAT repose sur l'identification d'agents spécifiques de chaque forme de l'enzyme ou d'inhibiteurs partiels pour éviter notamment des effets néfastes possibles résultant de l'inhibition totale de l'ACATl [26-28].

\section{Inhibiteurs d'IBAT}

Déversés dans l'intestin au niveau du duodénum, les acides biliaires sont réabsorbés dans l'iléon grâce au transporteur IBAT (ileal sodium-dependent bile acid transporter) localisé dans la membrane apicale des entérocytes (Figure 3). Ce transporteur est une cible intéressante, car son inhibition permettrait d'augmenter l'élimination des acides biliaires de l'organisme et d'activer la transformation du cholestérol en ces composés. Différents inhibiteurs d'IBAT ont été étudiés chez l'animal et se sont révélés efficaces en tant qu'hypocholestérolémiants. Le S-8921 (Shionogi), qui réduit la cholestérolémie de $70 \%$ chez les rats, est entré en phase I de développement clinique [29]. Cependant, il faut savoir que l'augmentation de la concentration d'acides biliaires libres dans la lumière intestinale pourrait provoquer des effets secondaires mineurs (diarrhée), mais aussi le développement de maladies intestinales (cancer du côlon) [30].

\section{Inhibiteurs de CETP}

La CETP (cholesterol ester transfer protein) est une enzyme qui facilite le transfert des esters de cholestérol des HDL vers les VLDL et les LDL (Figure 3), favorisant ainsi la diminution du cholestérol-HDL et l'augmentation du cholestérol-LDL, ce qui suggère un rôle pro-athérogène. Cepen- 
dant, à l'heure actuelle, il n'existe pas de consensus sur le rôle de la CETP, celle-ci apparaissant anti- ou pro-athérogène selon les études [31, 32]. II est communément admis qu'une augmentation de cholestérol-HDL d'environ entraîne non seulement un effet anti-athéromateux, mais aussi hypocholestérolémiant, ce qui justifie la recherche active d'agonistes du LXR par l'industrie pharmaceutique. Cependant, outre le métabolisme du
$30 \%$ serait bénéfique visà-vis du risque de développement de l'athérosclérose. Actuellement, le seul produit qui permet une telle augmentation est la niacine (acide nicotinique) qui est mal tolérée. Afin de disposer d'autres produits qui augmentent le cholestérol-HDL de façon efficace, des inhibiteurs de CETP sont néanmoins développés. L'un d'eux, testé en phase clinique II, le JT-705 (Japan Tobacco) augmente le cholestérol-HDL de $34 \%$ [33]. II reste à démontrer que cette augmentation est associée à une diminution du risque cardiovasculaire.

\section{Activateurs d'ABCA1}

Le transporteur ABCA (adenosine triphosphatebinding cassette Al) assure l'efflux du cholestérol des cellules périphériques et participe ainsi à la formation des HDL (Figure 3). Il intervient en outre dans l'absorption intestinale du cholestérol [34]. Au niveau de la lésion athéromateuse, $A B C l$ prévient l'accumulation du cholestérol dans les macrophages. Son expression est augmentée par les agonistes du récepteur nucléaire LXR (liver $X$ receptor). L'activité anti-athéromateuse d'un agoniste du LXR, le GW3965 (GlaxoSmithKline), a été montrée chez l'animal [35]. Chez l'animal, l'activation du LXR

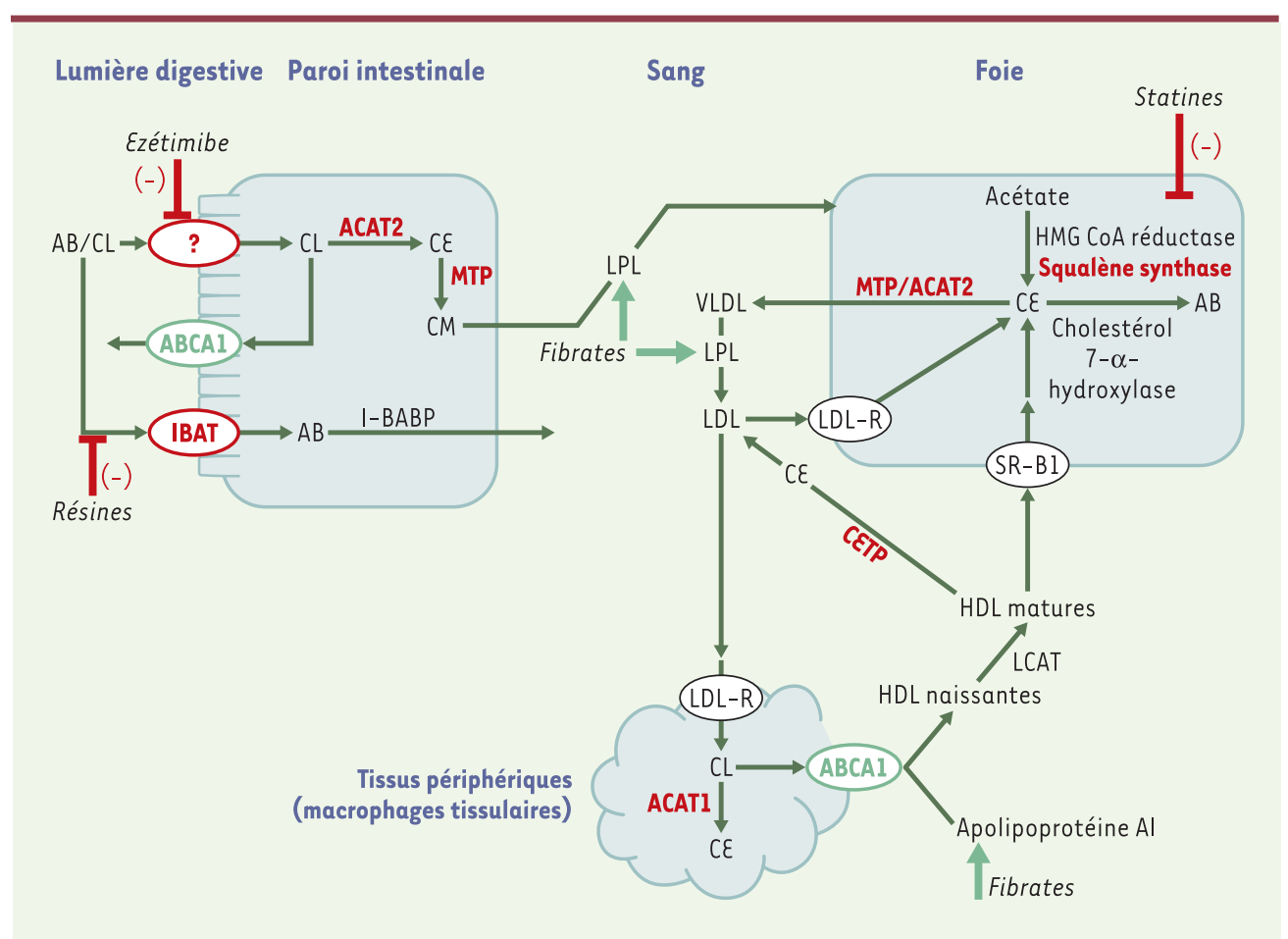

Figure 3. Métabolisme du cholestérol et cibles moléculaires des médicaments contre l'athérosclérose (existants ou en développement). Les cibles moléculaires visées par de nouvelles molécules médicamenteuses sont indiquées en vert (cibles qu'il faudrait activer) ou en rouge (cibles qu'il faudrait inhiber). Dans l'intestin, le cholestérol solubilisé par les acides biliaires $(A B)$ est absorbé par les entérocytes grâce à un ou plusieurs transporteurs du cholestérol inconnus à ce jour. Le cholestérol libre $(\mathrm{CL})$ absorbé est transformé en cholestérol estérifié $(C \varepsilon)$ par l'enzyme ACAT2 (acyl-coenzyme A:cholesterol acyltransferase 2) et incorporé dans les chylomicrons (CM) par l'action de la MTP (microsomal transfer protein). Le cholestérol libre pourrait aussi être expulsé des entérocytes vers la lumière intestinale par la protéine $\mathrm{ABCAl}$ (adenosine triphosphate-binding cassette Al). La réabsorption des acides biliaires par l'intestin se fait grâce au transporteur membranaire IBAT (ileal sodiumdependent bile acid transporter) et une protéine cytosolique I-BABP (ileal bile acid binding protein) $(\rightarrow)$. Dans le sang, la lipoprotéine lipase (LPL) hydrolyse les triglycérides portés par les chy- $(\rightarrow) \mathrm{m} / \mathrm{s}$ 2004, n' 1 , p. 73 lomicrons et libère les acides gras pour les tissus. Le foie est le site de synthèse du cholestérol dont l'étape limitante est catalysée par l'enzyme 3-hydroxy-3-méthylglutaryl coenzyme A (HMG CoA) réductase. Le foie est aussi le site exclusif de la transformation du cholestérol en $A B$ dont l'enzyme limitante est la cholestérol 7- $\alpha$ hydroxylase. Le foie utilise le cholestérol pour l'assemblage des VLDL (very low density lipoprotein) à l'aide de I'ACAT2 et de la MTP. Dans la circulation, les VLDL sont transformées en LDL (low density lipoprotein), dont le rôle est d'apporter le cholestérol aux tissus périphériques qui captent les LDL grâce à leur récepteur (LDL-R). Dans les tissus, le cholestérol peut être stocké sous forme d'esters (CE) grâce à l'enzyme ACATl, ou son excès peut être transporté à l'extérieur des cellules par des protéines telle que le transporteur ABCAl pour former des HDL (high density lipoprotein). Les HDL transportent le cholestérol excédentaire des tissus vers son site d'élimination (foie). Dans la circulation les HDL sont remodelées grâce aux différentes enzymes dont la LCAT (lecithin cholesterol acyltransferase) et la CETP (cholesterol ester transfer protein). Un des récepteurs via lesquels les HDL délivrent le cholestérol au foie est le SR-B1 (scavenger receptor class B type 1). Les principaux médicaments contre l'athérosclérose sont les statines (inhibiteurs d'HMG CoA réductase), les fibrates (baisse des triglycérides plasmatiques), et les résines (inhibiteurs de réabsorption des acides biliaires). 
cholestérol, le LXR contrôle également celui des acides gras. Chez les animaux traités, ses agonistes induisent une hypertriglycéridémie. Bien qu'éventuellement transitoire, celle-ci représente un effet secondaire indésirable vis-à-vis du risque de développement de l'athérosclérose [36]. Actuellement, aucun agoniste du LXR n'est entré en phase clinique de développement [20].

\section{Activateurs des récepteurs nucléaires PPAR}

Les PPAR contrôlent le métabolisme des lipides et du glucose. Leurs activateurs sont déjà utilisés en pratique médicale pour traiter les dyslipidémies (fibrates, agonistes du PPAR $\alpha$ ) et le diabète de type II (thiazolidinediones, agonistes du PPAR $\gamma$ ). Les agonistes des PPAR $\alpha$ et $\gamma$ influencent aussi le processus d'athérogenèse par leur action anti-inflammatoire, ce qui pourrait expliquer leur effet anti-athéromateux observé chez l'animal. En raison de ce potentiel thérapeutique, de nouveaux agonistes du PPAR $\gamma$ sont recherchés, car ils pourraient réduire l'athérogenèse chez le patient diabétique. Des activateurs mixtes PPAR $\alpha / \gamma$ sont également étudiés, car ils combineraient des effets sur les dyslipidémies, le diabète et l'athérome. De nombreux produits de ce type identifiés actuellement par les firmes pharmaceutiques se révèlent athéroprotecteurs chez l'animal. Cependant, des études cliniques sont nécessaires pour déterminer si ces produits ont une

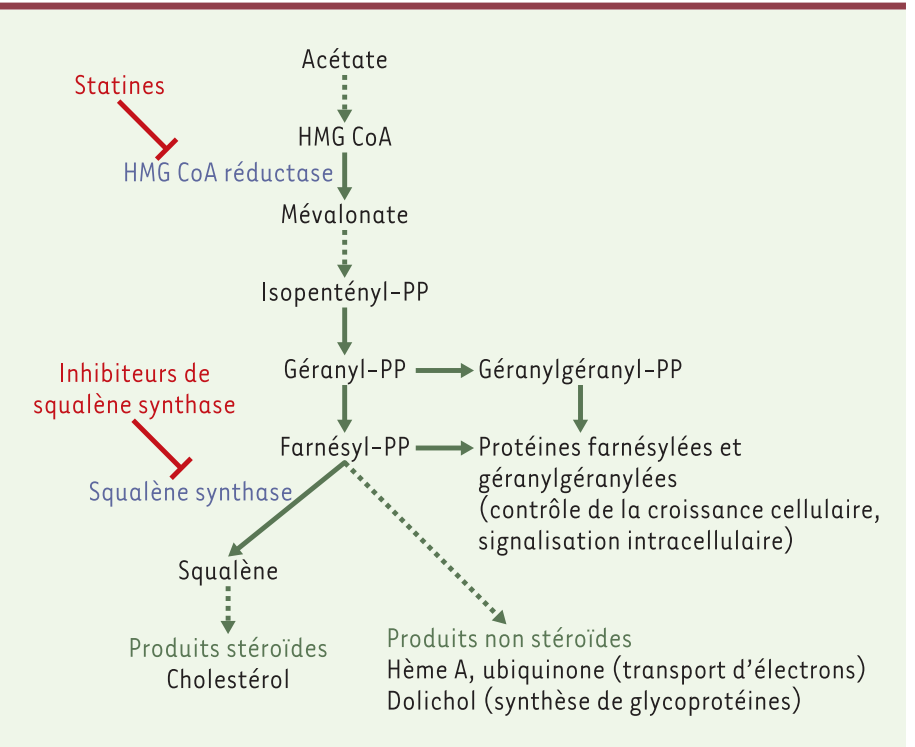

Figure 4. Voie de synthèse des isoprénoïdes. Le point de départ de cette voie est l'acétate qui est converti grâce à plusieurs enzymes, dont la 3-hydroxy-3méthylglutaryl coenzyme A (HMG CoA) réductase, en farnésyl-pyrophosphate. Ce dernier est le point d'embranchement vers la synthèse de produits stéroïdes (cholestérol) ou de produits non stéroïdes (ubiquinone, dolichols, hème A...). La squalène synthase catalyse la première étape de la voie de synthèse spécifique des stéroïdes. PP: pyrophosphate. action protectrice contre les événements cardiovasculaires fatals chez I'homme, d'autant que les agonistes du PPAR $\gamma$ pourraient exercer des effets non désirés sur les triglycérides et les $\operatorname{LDL}[37,38]$. Les résultats d'essais de phase III menés actuellement avec l'agoniste PPAR $\alpha / \gamma$ KRP-297 (Kyorin/Merck) pourraient donner en partie la réponse.

Les produits décrits ci-dessus, avec leurs effets souhaités sur le métabolisme lipidique mais aussi leurs effets non désirés, représentent autant de pistes qui continuent à être explorées chez l'animal et dans la mesure du possible chez l'homme, pour déterminer lesquels seront utilisables dans la prévention de l'athérosclérose chez les patients.

Afin de développer des médicaments athéroprotecteurs, d'autres cibles moléculaires sont également à l'étude. C'est le cas des molécules d'adhérence comme VCAM-1 (vascular cell adhesion molecule-1) ou de facteurs immunitaires comme MCP-1 (monocyte chemoattractant protein 1) dont l'expression est diminuée par le produit AGI-1067 (AtheroGenics) [39]. La modification d'activité de récepteurs scavengers de classe $\mathrm{B}$ (SR-B1 - scavenger receptor class B type 1, CD36 - cluster of differentiation 36) pourrait également constituer une stratégie de traitement de l'athérosclérose; toutefois, aucun médicament n'a encore été développé dans ce domaine. Enfin, l'inhibition de l'oxydation des LDL dans la paroi vasculaire est également envisagée. Cependant, l'administration d'antioxydants naturels (vitamine $\varepsilon$, caroténoïdes) s'est révélée inefficace dans la réduction du risque cardiovasculaire chez l'homme [40].

\section{Conclusions}

Bien que de grands progrès aient été réalisés ces 20 dernières années pour prévenir l'athérosclérose, celle-ci reste toujours la principale cause de mortalité dans les pays industrialisés. Les stratégies actuelles, qui visent avant tout à modifier les paramètres lipidiques plasmatiques, n'apportent pas de solution thérapeutique à l'ensemble des patients. Des études plus fines des mécanismes physiologiques impliqués sont donc à poursuivre pour proposer de nouvelles approches médicamenteuses. Des médicaments anti-athéromateux qui empêcheraient l'accumulation de cholestérol ou diminueraient l'inflammation directement au niveau de la paroi vasculaire pourraient réduire efficacement l'athérogenèse. L'intérêt de nouveaux produits hypocholestérolémiants, qui pourront difficilement concurrencer les statines, réside dans la possibilité de les administrer avec ces dernières, de façon à atteindre les valeurs recommandées de cholestérolémie et éviter la toxicité observée à des doses élevées de statines. Malgré leur complexité, le métabolisme du cholestérol et le mécanisme d'athérogenèse sont une source riche de cibles moléculaires étudiées pour développer de nouveaux médicaments contre l'athérosclérose. Les travaux qui en découlent devraient contribuer à diminuer l'impact négatif des maladies cardiovascu- 
laires sur la longévité, non seulement dans les pays industrialisés, mais également dans le reste du monde, car ces maladies n'ont pas de limites socio-économiques ou géographiques. $\diamond$

\section{REMERCIEMENTS}

Les auteurs souhaitent remercier Christian Guffroy de l'équipe Veille scientifique et concurrentielle de Fournier Pharma pour la mise à leur disposition des informations utiles à la rédaction de cet article, ainsi que Christiane Legendre et Patrick Dutartre pour la lecture critique du manuscrit.

\section{SUMMARY}

\section{Cholesterol metabolism modulators in future drug therapy}

for atherosclerosis

Atherosclerosis is a disease characterized by lipid accumulation in the vascular wall leading to myocardial infarction or stroke. Hypercholesterolemia is an important risk factor and current treatments are largely based on cholesterol lowering. In spite of proven efficacy of existing drugs, like statins, cardiovascular diseases still remain the most common cause of death in industrialised countries. Many new molecular targets are being studied to improve atherosclerosis treatment and reduce the number of deaths. The action on these targets could lead to a decrease of blood cholesterol levels or produce a direct antiatherosclerotic effect on the vascular wall. A cholesterol lowering effect could be achieved by reducing cholesterol synthesis (squalene synthase inhibitors), intestinal cholesterol absorption as well as intestinal and liver lipoprotein secretion (microsomal transfer protein inhibitors, acyl-coenzyme A-cholesterol acyltransferase inhibitors) or by increasing fecal excretion of bile acids (ileal sodium-dependent bile acid transporter inhibitors). An anti-atherosclerotic effect on the vascular wall could be achieved by reducing the inflammation via activation of peroxisome proliferator activated receptors, or, more particularly, could consist of decreased expression of adhesion molecules and chemoattractant proteins. Increasing the adenosine triphosphate-binding cassette $\mathrm{Al}$ protein and inhibiting acylcoenzyme A:cholesterol acyltransferase 1 activity could slow down formation of foam cells, which are a hallmark of atherosclerosis. Finally, the cholesterol fraction carried by high density lipoproteins, which is inversely correlated to cardiovascular risk, could be increased by cholesterol ester transfer protein inhibitors. All of these new classes of compounds are currently studied by pharmaceutical companies and are in different phases of development (preclinical or clinical). $\Delta$

\section{RÉFÉRENCES}

1. Berliner JA, Navab M, Fogelman AM, et al. Atherosclerosis: Basic mechanisms. Oxidation, inflammation, and genetics. Circulation 1995; 91: 2488-96.

2. Libby P. Changing concepts of atherogenesis. J Intern Med 2000; 247: 349-58.

3. Lusis AJ. Atherosclerosis. Nature 2000; 407: 233-41.

4. Assmann G, Cullen P, Jossa F, et al. Coronary heart disease: Reducing the risk: The scientific background to primary and secondary prevention of coronary heart disease. A worldwide view. International Task force for the Prevention of Coronary Heart disease. Arterioscler Thromb Vasc Biol 1999; 19: 1819-24.

5. Braunwald $\varepsilon$. Shattuck lecture - cardiovascular medicine at the turn of the millennium: Triumphs, concerns, and opportunities. N Engl/ Med 1997; 337: 1360-9.

6. Russell DW. Cholesterol biosynthesis and metabolism. Cardiovasc Drugs Ther 1992 ; 6: $103-10$.

7. Ros $\varepsilon$. Intestinal absorption of triglyceride and cholesterol. Dietary and pharmacological inhibition to reduce cardiovascular risk. Atherosclerosis 2000 ; $151: 357-79$.
8. Bellosta S, Bernini F, Ferri N, et al. Direct vascular effects of HMG-CoA reductase inhibitors. Atherosclerosis 1998; 137: S101-9.

9. Davignon J. Advances in drug treatment of dyslipidemia: Focus on atorvastatin. Can J Cardiol 1998; 14 (suppl B) : 28B-38.

10. Farnier M, Davignon J. Current and future treatment of hyperlipidemia: The role of statins. Am J Cardiol 1998; 82: 3J-10.

11. Ross SD, Allen IE, Connelly J $\varepsilon$, et al. Clinical outcomes in statin treatment trials: A meta-analysis. Arch Intern Med 1999; 159: 1793-802.

12. Staels B, Dallongeville J, Auwerx J, et al. Mechanism of action of fibrates on lipid and lipoprotein metabolism. Circulation 1998; 98: 2088-93.

13. van Heek M, Davis H. Pharmacology of ezetimibe. Eur Heart J $2002 ; 4$ (suppl) : J5-8.

14. Bruckert $\varepsilon$, Giral P, Tellier P. Perspectives in cholesterol-lowering therapy. The role of ezetimibe, a new selective inhibitor of intestinal cholesterol absorption. Circulation 2003; 107 : 3124-8.

15. Sleight P. Angiotensin II and trials of cardiovascular outcomes. Am J Cardiol 2002; 89: 11A-7.

16. Evans $M$, Roberts $A$, Rees $A$. The future direction of cholesterol-lowering therapy. Curr Opin Lipidol 2002; 13: 663-9.

17. Brousseau ME, Schaefer $\varepsilon$ J. New targets for medical treatment of lipid disorders. Curr Atheroscler Rep 2002; 4 : 343-9.

18. Brown WV. Therapies on the horizon for cholesterol reduction. Clin Cardiol 2001; 24: III24-7.

19. Thompson GR, Naoumova RP. New prospects for lipid-lowering drugs. Exp Opin Invest Drugs 1998; 7: 715-27.

20. Pharmaproject. Richmond, Surrey (UK): PJB Publications, 2003.

21. Ugawa T, Kakuta H, Moritani H, et al. YM-53601, a novel squalene synthase inhibitor, reduces plasma cholesterol and triglyceride levels in several animal species. BrJ Pharmacol 2000; 131: 63-70.

22. Chevy F, Illien F, Wolf C, Roux C. Limb malformations of rat fetuses exposed to a distal inhibitor of cholesterol biosynthesis. J Lipid Res 2002; 43: 1192-200.

23. Chang G, Ruggeri RB, Harwood HJ, Jr. Microsomal triglyceride transfer protein (MTP) inhibitors: Discovery of clinically active inhibitors using high-throughput screening and parallel synthesis paradigms. Curr Opin Drug Discov Dev 2002; 5: 562-70.

24. Shiomi M, Ito T. MTP inhibitor decreases plasma cholesterol levels in LDL receptordeficient WHHL rabbits by lowering the VLDL secretion. Eur J Pharmacol 2001; 431: 127-31.

25. Nicolosi RJ, Wilson TA, Krause BR. The ACAT inhibitor, $\mathrm{Cl}-1011$ is effective in the prevention and regression of aortic fatty streak area in hamsters. Atherosclerosis $1998 ; 137: 77-85$

26. Accad M, Smith SJ, Newland DL, et al. Massive xanthomatosis and altered composition of atherosclerotic lesions in hyperlipidemic mice lacking acyl $\mathrm{CoA}$ : Cholesterol acyltransferase 1.J Clin Invest 2000; 105: 711-9.

27. Willner EL, Tow B, Buhman KK, et al. Deficiency of acyl CoA: Cholesterol acyltransferase 2 prevents atherosclerosis in apolipoprotein $\varepsilon$-deficient mice. Proc Natl Acad Sci USA 2003; 100 : 1262-7.

28. Brewer HB Jr. The lipid-laden foam cell: An elusive target for therapeutic intervention. J Clin Invest 2000; 105: 703-5.

29. Ichihashi T, Izawa M, Miyata K, et al. Mechanism of hypocholesterolemic action of S-8921 in rats: S-8921 inhibits ileal bile acid absorption. J Pharmacol Exp Ther $1998 ; 284: 43-50$.

30. Izzat NN, Deshazer ME, Loose-Mitchell DS. New molecular targets for cholesterollowering therapy.J Pharmacol Exp Ther 2000; 293: 315-20.

31. Zhong S, Sharp DS, Grove JS, et al. Increased coronary heart disease in JapaneseAmerican men with mutation in the cholesteryl ester transfer protein gene despite increased HDL levels. J Clin Invest 1996; 97 : 2917-23.

32. Okamoto $\mathrm{H}$, Yonemori $\mathrm{F}$, Wakitani $\mathrm{K}$, et al. A cholesteryl ester transfer protein inhibitor attenuates atherosclerosis in rabbits. Nature 2000; 406: 203-7.

33. de Grooth GJ, Kuivenhoven JA, Stalenhoef AF, et al. Efficacy and safety of a novel cholesteryl ester transfer protein inhibitor, JTT-705, in humans: A randomized phase II dose-response study. Circulation 2002; 105: 2159-65.

34. Repa JJ, Turley SD, Lobaccaro JA, et al. Regulation of absorption and ABClmediated efflux of cholesterol by RXR heterodimers. Science 2000; 289: 1524-9.

35. Joseph SB, McKilligin $\varepsilon$, Pei L, et al. Synthetic LXR ligand inhibits the development of atherosclerosis in mice. Proc Natl Acad Sci USA 2002; 99: 7604-9.

36. Lund $\varepsilon G$, Menke JG, Sparrow CP. Liver X receptor agonists as potential therapeutic agents for dyslipidemia and atherosclerosis. Arterioscler Thromb Vasc Biol 2003; 23: 1169-77.

37. Barbier 0, Torra IP, Duguay Y, et al. Pleiotropic actions of peroxisome proliferatoractivated receptors in lipid metabolism and atherosclerosis. Arterioscler Thromb Vasc Biol 2002; 22: 717-26.

38. Klappacher GW, Glass CK. Roles of peroxisome proliferator-activated receptor gamma in lipid homeostasis and inflammatory responses of macrophages. Curr Opin Lipidol 2002; 13: 305-12.

39. Meng CQ, Somers PK, Rachita CL, et al. Novel phenolic antioxidants as multifunctional inhibitors of inducible VCAM- 1 expression for use in atherosclerosis. Bioorg Med Chem Lett 2002; 12: 2545-8.

40. Mashima R, Witting PK, Stocker R. Oxidants and antioxidants in atherosclerosis. Curr Opin Lipidol 2001 12: $411-8$.

\section{TIRÉS À PART}

S. Morozova 